\title{
Strong pinning of triple junction migration for robust high strain nanostructures
}

\author{
Yu, Tianbo; Hughes, Darcy A.
}

Published in:

Philosophical Magazine

Link to article, DOI:

$10.1080 / 14786435.2018 .1562282$

Publication date:

2019

Document Version

Peer reviewed version

Link back to DTU Orbit

Citation (APA):

Yu, T., \& Hughes, D. A. (2019). Strong pinning of triple junction migration for robust high strain nanostructures. Philosophical Magazine, 99(7), 869-886. https://doi.org/10.1080/14786435.2018.1562282

\section{General rights}

Copyright and moral rights for the publications made accessible in the public portal are retained by the authors and/or other copyright owners and it is a condition of accessing publications that users recognise and abide by the legal requirements associated with these rights.

- Users may download and print one copy of any publication from the public portal for the purpose of private study or research.

- You may not further distribute the material or use it for any profit-making activity or commercial gain

- You may freely distribute the URL identifying the publication in the public portal

If you believe that this document breaches copyright please contact us providing details, and we will remove access to the work immediately and investigate your claim. 
Strong pinning of triple junction migration for robust high strain nanostructures

Tianbo Yu*a and Darcy A. Hughes ${ }^{b}$

${ }^{a}$ Department of Mechanical Engineering, Technical University of Denmark, 2800 Kgs. Lyngby, Denmark

${ }^{b}$ Fremont, CA 94539, USA

*Corresponding author:

Tel:

(45) 45254889

Email: $\quad$ tiyu@mek.dtu.dk

ORCID: $\quad$ 0000-0001-9525-9354

Address: $\quad$ Produktionstorvet

Building 425 Room 213

2800 Kgs. Lyngby, Denmark 


\section{Strong pinning of triple junction migration for robust high strain nanostructures}

The universality of a key recovery mechanism: triple junction migration in high strain nanostructures is revealed herein. This migration is the only means to uniformly coarsen deformed lamellar microstructures. Migration of medium to high angle geometrically necessary boundaries at triple junctions is resisted by strong pinning phenomena. Pinning by low angle dislocation boundaries is the novel mechanism that greatly adds to the solute drag of these higher angle boundaries during migration at triple junctions. Solutes furthermore cause a significant increase in the dislocation density of the low angle boundaries formed during deformation and thus greatly enhance the observed pinning. Boundary pinning by dislocation boundaries and solute drag is analysed for deformed $\mathrm{Ni}$ of different purities via in and ex situ electron microscopy. A kinetic model is utilized to obtain activation energies that quantitatively demonstrate the strength of this pinning. A new strategy for achieving robust nanostructured metals is developed based on solute and dislocation pinning of triple junction migration - a universal recovery mechanism in deformed lamellar microstructures.

Keywords: Deformation microstructure; Triple junction migration; Recovery kinetics; Solute drag; Dislocation pinning; Activation energy

\section{Introduction}

Structural refinement of metals to the average nanometre dimension of $5 \mathrm{~nm}$ can be achieved by plastic deformation to extreme strain [1]. To reach and maintain the finest dimension, recovery and recrystallization must be supressed during both processing and storage. Recovery is a precursor to recrystallization so that both dynamic and static recovery mechanisms need to be identified and quantitatively analysed to best design the chemical composition and microstructure of advanced materials. The formation and removal of high angle (grain) boundaries is a key issue in the quest for ultrafine structural metals with very high strength and formability.

Triple junction migration is a novel recovery mechanism that removes boundaries during both plastic deformation and annealing, previously shown in Al [2-4]. These junctions, each formed by three lamellar boundaries were named Y-junctions based on their origin and morphology [3] (see also Fig. 1). In contrast to commonly known recovery mechanisms, Y-junction migration occurs in fine lamellar microstructures, i.e. microstructures formed by high monotonic strain. This migration increases lamellar boundary spacing via the 
local migration of lamellar boundaries at their Y-junctions, while maintaining a lamellar morphology that may very gradually transform into an equiaxed one $[2,3]$. Note that lamellar boundaries are defined as the geometrically necessary boundaries (GNBs) that enclose each lamella [5]. The underlying mechanisms that pin and interact with this migration in terms of the dislocation structure and solutes need to be understood to counteract coarsening and promote refinement.

To explore Y-junction migration as a universal recovery mechanism, a new experimental test was designed utilizing Ni with different solute contents, since Ni sits among the transition metals in the periodic table and has widely different properties compared to Al. Nickel differs substantially from Al in melting point (1455 vs. $\left.660^{\circ} \mathrm{C}\right)$, stacking fault energy $\left(0.125\right.$ vs. $\left.0.166 \mathrm{~J} / \mathrm{m}^{2}\right)$ and elastic properties (e.g. shear modulus of 79 vs. $26 \mathrm{GPa}$ ), all of which strongly affect thermally activated processes. The deformation microstructure and texture are similar in both heavily cold-rolled materials [5-7], but the structure in $\mathrm{Ni}$ is twice finer with a much higher stored energy. Stabilizing Ni to increase refinement thus presents a challenge. Herein mechanisms that stabilize the structure and alter the kinetics of $\mathrm{Y}$-junction migration are investigated based on the hypothesis that there is a strong link between the characteristics of the deformed microstructure and the mechanisms and kinetics of recovery and recrystallization.

\section{Materials and methods}

$\mathrm{Ni}$ of two purities (99.97\%, $3 \mathrm{~N}$ and $99.5 \%, 2 \mathrm{~N}$ ) was chosen since recovery and recrystallization are strongly affected by small amounts of impurities [8,9]. The commonly used solutes Fe and $\mathrm{Mn}$ are the principal impurities (Table 1). Both materials were deformed by accumulative roll bonding (ARB) to 6 cycles $\left(\varepsilon_{\mathrm{vM}}=4.8\right)$ at room temperature with lubrication (see [10] for details). The final thickness of the deformed material was 1 $\mathrm{mm}$, and particles were present in the bonding interfaces. After deformation, the $2 \mathrm{~N} \mathrm{Ni}$ was annealed between 300 and $700{ }^{\circ} \mathrm{C}$, whereas the $3 \mathrm{~N} \mathrm{Ni}$ was annealed between 100 and $400^{\circ} \mathrm{C}$.

After annealing, both Vickers hardness and microstructures were investigated in the longitudinal section, containing the rolling direction (RD) and the normal direction (ND). Samples were mechanically polished followed by electropolishing [8]. Vickers hardness tests were carried out with a load of $200 \mathrm{~g}$ and a holding time of $10 \mathrm{~s}$, and at least 6 measurements were performed for each state. Due to the throughthickness microstructural and textural variations in ARB-deformed samples [11], microstructural characterizations were carried out in the vicinity of the thickness centre.

For electron backscatter diffraction (EBSD) analysis, step sizes between 15 and $50 \mathrm{~nm}$ were chosen. EBSD data were collected and processed using HKL Channel 5 software, and a critical angle of $1.5^{\circ}$ was used in boundary detection. Thin foils for transmission electron microscopy (TEM) analyses were prepared using a 
modified window technique [12], and the experiment was carried out in a JEM 2100 transmission electron microscope, which was operated at $200 \mathrm{kV}$. For the in situ TEM observations, samples were heated at a heating rate of $0.2-0.4{ }^{\circ} \mathrm{C} / \mathrm{s}$, and the microstructural changes were recorded by a TVIPS FastScan camera with a maximum frame rate of $12 \mathrm{fps}$ at full resolution $(1024 \times 1024)$.

\section{Results}

\subsection{Deformation microstructure}

The deformation microstructure of both purities of $\mathrm{Ni}$ is composed of fine lamellae that are universally observed following high strain monotonic deformation in fcc (face-centred cubic) and bcc (body-centred cubic) metals [6], e.g. Figs. 1a-c. Lamellae are enclosed by medium to high angle GNBs whose interiors are further subdivided by low angle incidental dislocation boundaries (IDBs) and dislocations (Figs. 1-3). The IDBs are randomly inclined about the ND and connect directly to the lamellar GNBs. These two types of boundaries are created by the deformation; i.e. they are deformation-induced. Both types are also rotation boundaries that separate volumes with different crystal orientations.

Boundary spacings are given in Table 2 for lamella measured along the ND by TEM, EBSD and electron channelling contrast (ECC), and for IDBs measured parallel to the lamella. Also tabulated are fractions of high angle boundaries $\left(>15^{\circ}\right)$ and rolling texture components. The average misorientation angle of the IDBs is between 1.5 and $3^{\circ}$. The average length to thickness aspect ratio of the thin lamella is $\sim 10$. All aspects of $2 \mathrm{~N} \mathrm{Ni}$ deformation microstructure are remarkably finer than that of $3 \mathrm{~N} \mathrm{Ni}$, demonstrating a strong effect of solutes while suggesting a much higher stored energy and driving force for recovery and recrystallization in $2 \mathrm{~N} \mathrm{Ni}$.

An abundance of $\mathrm{Y}$-junctions was observed in both $2 \mathrm{~N}$ and $3 \mathrm{~N} \mathrm{Ni}$. Of special note is the observation that an IDB is almost always observed externally attached to the $\mathrm{Y}$-junctions. This measured proximity is much closer than a random distribution. Boundary dislocations in IDBs may connect through their common GNB to the network dislocations in IDBs within the middle lamella of a Y-junction (Fig. 3). Connections between IDB dislocations on one side of a GNB to IDB dislocations on the other side may occur via extrinsic and intrinsic GNB dislocations, see e.g. [13].

This deformation structure typically contains some coarse slip localized in bands of micro shear (see section 3.4 Fig. 8a). These bands sharply intersect lamella and play an active role in the dynamic creation of new $Y$-junctions that subsequently migrate and annihilate. See for example the special configurations of $Y$ junctions in the deformed microstructure in Fig. 4, where many pairs of lamellae (two examples are shaded) 
have a similar orientation while forming high angle boundaries with neighbouring lamellae. This configuration occurs when a lamella is pinched by shear thereby creating a pair of $\mathrm{Y}$-junctions that migrate away from each other during deformation, i.e. dynamic Y-junction migration has taken place during processing (see also [14] for an example in Al). Y-junction migration is responsible for the net dynamic loss of lamellar GNBs that accompanies refinement during deformation.

\subsection{Hardness during recovery and recrystallization}

The hardness of $2 \mathrm{~N} \mathrm{Ni}$ is higher than that of $3 \mathrm{~N} \mathrm{Ni}$ mainly due to its much finer microstructure. Despite a higher stored energy, $2 \mathrm{~N} \mathrm{Ni}$ is thermally more stable, and its recrystallization temperature is about $300^{\circ} \mathrm{C}$ higher than that of $3 \mathrm{~N} \mathrm{Ni} \mathrm{(Fig.} \mathrm{5),} \mathrm{indicating} \mathrm{a} \mathrm{strong} \mathrm{solute} \mathrm{effect} \mathrm{on} \mathrm{the} \mathrm{thermal} \mathrm{stability} \mathrm{as} \mathrm{well} \mathrm{as} \mathrm{structural}$ refinement. A high recrystallization temperature gives rise to a large recovery window in $2 \mathrm{~N} \mathrm{Ni}$; in situ TEM observations were thus performed for $2 \mathrm{~N} \mathrm{Ni}$.

\subsection{Y-junction migration in situ and ex situ studies}

During in situ annealing of $2 \mathrm{~N} \mathrm{Ni}$ inside a transmission electron microscope, the migration of $\mathrm{Y}$-junctions was observed and recorded. During recovery, boundary migration was observed only locally at $\mathrm{Y}$-junctions where the GNBs have a high local curvature (Fig. 6); i.e. generalized boundary migration did not occur anywhere else prior to recrystallization. Fig. 6a illustrates the downward migration as the temperature increases of a Yjunction terminating a thin lamella of black contrast. Migration of the $\mathrm{Y}$-junction shortened the middle thin lamella, replaced two lamellar GNBs by one and increased the average lamellar boundary spacing, while keeping the general lamellar morphology. When the temperature was further increased, more $\mathrm{Y}$-junctions migrated, e.g. Fig. 6b, where the arrowed Y-junction migrated upwards for a long distance as the temperature increased from 350 to $400^{\circ} \mathrm{C}$. In most cases, the removed/shortened lamellae were less than $60 \mathrm{~nm}$ thick in the ND, much thinner than the average lamellar boundary spacing shown in Table 2. The IDBs in neighbouring lamellae exhibit a strong pinning effect, retarding Y-junction migration. Junctions visibly paused and significantly slowed their migration when they reached an IDB. Migrating junctions in Fig. 6 ultimately stop their migration at IDBs.

The uniform coarsening of the recovered matrix was further investigated by ex situ EBSD during annealing. For example, Fig. 7 shows the raw EBSD data of a small area in $3 \mathrm{~N} \mathrm{Ni}$ before and after annealing for 1 hour at $200^{\circ} \mathrm{C}$. The black arrows indicate three examples of $\mathrm{Y}$-junction motion. 


\subsection{Microstructural coarsening in bulk samples}

In situ TEM observations on thin foils directly revealed the local mechanisms responsible for the coarsening pattern of the heavily deformed $2 \mathrm{~N} \mathrm{Ni}$. To study the global coarsening kinetics in the interior, bulk samples were annealed and investigated by TEM, EBSD and ECC. Measurements of the average lamellar GNB spacing demonstrated that the annealed microstructure (Figs. 8-10) developed a coarse lamella spacing compared to the as-deformed sample (Fig. 1a), and agreed with the coarsening pattern revealed by the in situ TEM observations. After annealing at $400{ }^{\circ} \mathrm{C}$ (up to $120 \mathrm{~h}$ ), $450^{\circ} \mathrm{C}$ (up to $17 \mathrm{~h}$ ) and $500^{\circ} \mathrm{C}$ (up to $4 \mathrm{~h}$ ), the microstructure coarsened and examples are shown in Fig. 8. No recrystallization was observed in samples annealed at $400{ }^{\circ} \mathrm{C}$. Nucleation of recrystallization was first observed after annealing at $450{ }^{\circ} \mathrm{C}$ for $7 \mathrm{~h}$ and at $500{ }^{\circ} \mathrm{C}$ for 10 minutes, where the lamellar GNB spacing of the matrix was increased from about 0.11 to 0.16 $\mu \mathrm{m}$ as measured by EBSD. The nuclei are sparse and small, and their volume fraction is less than $1 \%$ (Fig. 9) except for one annealing condition $\left(500^{\circ} \mathrm{C}\right.$ for $4 \mathrm{~h}$ ), where the volume fraction of nuclei has increased to about $5 \%$. Average lamellar GNB spacings measured in the recovered matrix are plotted in Fig. 10b.

The $3 \mathrm{~N} \mathrm{Ni}$ in contrast is thermally less stable with recovery and recrystallization occurring already during anneals between 200 and $300^{\circ} \mathrm{C}$ (e.g. Fig. 11). After annealing, the average lamellar GNB spacing of the recovered matrix increased from about 0.16 to $0.18 \mu \mathrm{m}$ as measured by EBSD. A number of recrystallization nuclei, typically 1-4 $\mu \mathrm{m}$ in size and of Cube orientation, were also formed simultaneously. Y-junction migration was confirmed as the cause of uniform coarsening by ex situ EBSD during annealing at $200^{\circ} \mathrm{C}$ (e.g. Fig. 7).

\subsection{Effect of lamellar coarsening and annealing on the IDBs}

During annealing, IDBs maintained a spacing and density similar to the as-deformed sample due to the geometrical continuity of rotation boundaries. The overall dislocation density within lamella is thus relatively unchanged by Y-junction migration, even though some dislocations were observed by in situ TEM to rearrange and annihilate. These observations match the much higher hardness in $2 \mathrm{~N}$ compared to $3 \mathrm{~N}$ Ni for similar annealed lamella spacing (Fig. 5).

\subsection{Coarsening kinetics}

Experimental observations of a lamellar microstructure and recovery by $\mathrm{Y}$-junction migration in $\mathrm{Ni}$ are similar to those in Al. The coarsening kinetics model for deformed microstructures [15] is applied herein and assumes that the driving force for coarsening comes from the stored energy that is proportional to the area of deformation induced boundaries per unit volume. The model considers the increase of the apparent activation energy during coarsening. Following a classical recovery rate equation [16], it can be expressed as: 


$$
\frac{d D}{d t}=k_{1} D \exp \left(\frac{k_{2}}{D T}\right)
$$

By the aid of exponential integrals, the integrated form can be expressed as:

$$
\operatorname{Ei}\left(-\frac{k_{2}}{D T}\right)-E i\left(-\frac{k_{2}}{D_{0} T}\right)=-k_{1} t
$$

where $E_{\mathrm{i}}()$ is the exponential integral of the quantity inside the bracket, $D$ is the boundary spacing, $D_{0}$ is the boundary spacing before annealing, $T$ is the annealing temperature, $t$ is the annealing time, $k_{2}$ is a constant to be fitted, and $k_{1}$ is temperature dependent written as:

$$
k_{1}=K_{0} \exp \left(-\frac{Q_{0}}{R T}\right)
$$

where $R$ is the gas constant, $K_{0}$ is a constant to be fitted, and $Q_{0}$ is the extrapolated activation energy at the end of recovery. The three fitting parameters $K_{0}, Q_{0}$ and $k_{2}$ are associated with the operative coarsening mechanisms.

Eq. (2) describes the continuous coarsening of a deformation microstructure during isothermal annealing, and is applied to $2 \mathrm{~N}$ Ni based on the lamella spacing (Fig. 10b). Consequently as shown in Fig. 12, $Q_{0}$ can be estimated based on Eq. (3) and the apparent activation energy $Q_{a p p}$ at any stage of coarsening can be estimated based on the following equation:

$$
Q_{a p p}=Q_{0}-\frac{k_{2} R}{D}
$$

The apparent activation energy increased from 260 to $300 \mathrm{~kJ} / \mathrm{mol}$ in the current experimental coarsening range $(0.11-0.18 \mu \mathrm{m})$ for $2 \mathrm{~N} \mathrm{Ni}$ (Fig. 12). The increase of the apparent activation energy by 40 $\mathrm{kJ} / \mathrm{mol}$ is the main cause of the dramatic decrease of the coarsening rate from the early stage where $D=0.11$ $\mu \mathrm{m}$ to a late stage where $D=0.18 \mu \mathrm{m}$.

Due to an early interference of recrystallization in $3 \mathrm{~N} \mathrm{Ni}$, such an analysis is not informative.

\subsection{Recrystallization kinetics}

For the $2 \mathrm{~N} \mathrm{Ni}$ samples a fully recrystallized microstructure was observed after anneals at either $550{ }^{\circ} \mathrm{C}$ for $16 \mathrm{~h}$ or $600{ }^{\circ} \mathrm{C}$ for $0.25 \mathrm{~h}$. The average grain size is about $4 \mu \mathrm{m}$ if twin boundaries are not considered, the fraction of 
high angle boundaries is over $90 \%$, and the microstructure contains $20 \%$ Cube texture component, $35 \%$ retained rolling texture components and $45 \%$ random texture components according to EBSD analysis. In contrast the $3 \mathrm{~N} \mathrm{Ni}$ samples recrystallized at much lower temperatures, so that after $220^{\circ} \mathrm{C}$ for $2.5 \mathrm{~h}$ recrystallization was almost complete. This recrystallization resulted in an average grain size of $10 \mu \mathrm{m}$, a high fraction of high angle boundaries (70\%), and a strong Cube texture (68\%).

The apparent activation energy for recrystallization was estimated by comparing the annealing time needed at different temperatures when half of the volume was recrystallized. The fraction of recrystallized grains following annealing was calculated based on the measured distance covered by recrystallized grains along random lines drawn on ECC images. That distance was then divided by the total random line length to obtain the fraction recrystallized. These measurements are shown in Fig. 13a for $2 \mathrm{~N}$ Ni annealed between 500 and $600{ }^{\circ} \mathrm{C}$ and in Fig. $13 \mathrm{C}$ for $3 \mathrm{~N} \mathrm{Ni}$ annealed between 200 and $240{ }^{\circ} \mathrm{C}$. When the apparent activation energy for recrystallization is assumed to be a constant, the recrystallization kinetics curves at different temperatures should follow the same master curve with only a shift in position as sketched in Figs. 13a and c. The apparent activation energy for recrystallization was estimated for each material by comparing the annealing time needed at different temperatures when half of the volume was recrystallized. These energies are $270 \mathrm{~kJ} / \mathrm{mol}$ for $2 \mathrm{~N} \mathrm{Ni}$ and $100 \mathrm{~kJ} / \mathrm{mol}$ for $3 \mathrm{~N}$ reflecting their different responses to annealing (Figs. 13b and d respectively $)^{1}$. These activation energies are comparable to that for concurrent uniform coarsening by $Y$ junction motion, suggesting a fierce competition.

\section{Discussion}

Recovery coarsening absent in low strain Ni [18] but reported for medium-high strain Ni [19] was demonstrated in Ni of two purities deformed by ARB to a high strain. Y-junction migration is identified herein as the dominant mechanism for recovery coarsening, in agreement with previous observations in heavily deformed Al [2,3]. The universality of the lamellar microstructure formed by GNBs and IDBs [6], and the agreement with respect to $\mathrm{Y}$-junction migration between materials with very different material properties indicates the universality of this recovery mechanism. The strong connection between the characteristics of

${ }^{1}$ It should be noted that the kinetics of recrystallization for $3 \mathrm{~N} \mathrm{Ni}$ at $220^{\circ} \mathrm{C}$ shown in (c) is slightly different from that reported in a recent recrystallization study of the same deformed $\mathrm{Ni}$ [17]. This difference may have its cause in heterogeneities introduced during deformation and therefore a larger experimental error in the estimated activation energy is expected in the $3 \mathrm{~N} \mathrm{Ni}$ compared to that in the $2 \mathrm{~N} \mathrm{Ni}$. 
the deformation microstructure and the mechanisms and kinetics of recovery and recrystallization for highly deformed structural metals is thus demonstrated for the first time.

\subsection{Y-junction migration}

$\mathrm{Y}$-junctions are present in the deformed microstructure and their migration does not require an incubation period. Y-junction migration starts at slightly lower activation energies than required by recrystallization. It is less affected by solute drag since solutes may deposit at the tailing boundary. Y-junction migration is further enhanced by the high local boundary curvature that depends on lamella spacing (see Fig. 1). Consequently, uniform coarsening by $\mathrm{Y}$-junction migration occurs earlier than recrystallization although both processes involve migration of medium/high angle boundaries. Thin lamellae are preferentially removed, reducing the skewness of the boundary spacing distribution from 1.6 to 1 (Fig. 14). This change is opposite to recrystallization, which produces large new grains that increase the skewness significantly [3].

\subsection{The effect of solutes/impurities on recovery versus recrystallization}

In the competition between uniform coarsening and recrystallization, the former can be enhanced and the latter suppressed via changes in basic materials properties and processing parameters. Solutes/impurities have a significant effect on increased structural refinement and in stabilizing the deformed microstructure against recovery and recrystallization [8,9]. The recrystallization temperature of $2 \mathrm{~N} \mathrm{Ni}$ was about $300^{\circ} \mathrm{C}$ higher than that of $3 \mathrm{~N} \mathrm{Ni}$. This increase gave $2 \mathrm{~N} \mathrm{Ni}$ a larger window for uniform coarsening. Uniform coarsening consumed half of the stored energy, which is assumed to be proportional to the boundary area per unit volume. The apparent activation energy for uniform coarsening increased from 260 to $300 \mathrm{~kJ} / \mathrm{mol}$, close to the energy for recrystallization $(270 \mathrm{~kJ} / \mathrm{mol})$. Both of them are consistent with the solute drag of $\mathrm{Fe}$ and $\mathrm{Mn}$ in the $\mathrm{Ni}$ matrix [20]. This similarity underpins the fierce competition between recovery and recrystallization via the migration of medium/high angle boundaries.

In contrast, the activation energy for recrystallization in $3 \mathrm{~N} \mathrm{Ni}$ is low ( $100 \mathrm{~kJ} / \mathrm{mol})$ due to a very low solute concentration, and similar to grain boundary self-diffusion, $123 \mathrm{~kJ} / \mathrm{mol}$ [21], indicating a similar barrier for $\mathrm{Ni}$ atoms jumping across and along the boundary. Comparably low activation energies are thus expected for Y-junction migration in $3 \mathrm{~N} \mathrm{Ni}$, since $\mathrm{Y}$-junction migration was observed to occur simultaneously to recrystallization. 


\subsection{Dislocation pinning of junction migration and recovery coarsening}

Unique to $\mathrm{Y}$-junction migration is the pinning effect of IDBs that retards migration. The pinning has its cause in the interaction between dislocations in low angle IDBs that attach to lamellar GNBs (see Fig. 3 and sections 3.1 and 3.3). Before a moving Y-junction can pass an IDB that IDB must be transferred to the new trailing GNB. The IDB dislocations attached to the GNB are transferred one by one slowly across the junction (see also [22]). In addition the transferred IDB must be lengthened to fill the gap left by the retreating GNB. More complex dislocation interactions and exchanges between the two types of boundaries may be necessary to preserve the geometric continuity of the rotation boundaries (e.g. [13]).

This pinning occurs regardless of solute content, but the presence of solutes greatly increases the density of dislocations in IDBs (see Fig. 2) and thus their pinning force. It has long been known that solutes and impurities impede dislocation motion during both dynamic and static recovery. The high density of IDBs and their significant retarding effect indicates that this pinning will be manifested in the kinetics of $\mathrm{Y}$-junction migration. While pinning is a subject for future work, a simple analysis is possible. The dislocation density between lamella were shown to remain fairly constant during annealing, thus their pinning effect may be treated as a constant in the coarsening model. This pinning is then embedded in the value of $K_{0}$ in Eq. (3); the calculated apparent activation energy applies to the IDB pinning herein. As a consequence the solute concentration affects not only solute drag of the boundaries but also the dislocation/IDB de-pinning mechanism.

\subsection{Comparison between $2 \mathrm{~N} \mathrm{Al}$ and $2 \mathrm{~N} \mathrm{Ni}$}

The annihilation and reorganization of dislocations are strongly dependent on the stacking fault energy and elastic properties. Compared to Al, static and dynamic recovery is therefore much more difficult in Ni resulting in IDB walls that are much less sharp and cells containing more interior dislocations (e.g. compare Figs. 1a, b and $\mathrm{c}$ as well as [5-7]. The finer microstructure in Ni provides a larger driving force [3] for Y-junction motion, but at the same time there is also a larger pinning effect [22] due to more IDBs and interior dislocations in $\mathrm{Ni}$.

The fact that there is less uniform coarsening in $\mathrm{Ni}$ than in $\mathrm{Al}$ is also strongly related to the earlier onset of recrystallization in $\mathrm{Ni}$ aided by its very high stored energy. At a purity of $99.5 \%$, nucleation of recrystallization in $\mathrm{Al}$ starts at a homologous temperature of 0.53 of the absolute melting temperature $\left(220^{\circ} \mathrm{C}\right)$ [15], whereas in $\mathrm{Ni}$ it starts at $0.42\left(450^{\circ} \mathrm{C}\right)$. If we compare the coarsening at the same homologous temperature of 0.42 , which means $120^{\circ} \mathrm{C}$ for Al, then their coarsening rates are indeed quite similar (Fig. 15). 


\subsection{Dynamic Y-junction migration}

Dynamic Y-junction migration is an important mechanism counter-balancing structural refinement during high strain [14]. The present study shows that solutes contribute greatly to refinement during deformation by retarding dynamic Y-junction migration, decreasing lamella GNB spacing and increasing the density of dislocations in IDBs, e.g. compare $2 \mathrm{~N}$ and $3 \mathrm{~N} \mathrm{Ni}$. The fine spacing of lamellar GNBs and IDBs provide a high driving force for recovery and recrystallization. Solutes counteract that driving force by segregating at both boundaries and dislocations. Solutes thereby stabilize IDBs as well as lamellar GNBs to pin Y-junction migration.

\section{Conclusion}

Uniform coarsening of lamellae was observed in heavily deformed and annealed $2 \mathrm{~N}$ and $3 \mathrm{~N}$ Ni preceding recrystallization. Thermally activated $\mathrm{Y}$-junction migration is the only means to uniformly coarsen deformed lamellar microstructures as seen herein and in agreement with previous observations in Al, suggesting the universality of this recovery mechanism. Loss of medium to high angle lamellar GNBs via Y-junction migration links the deformation microstructure to the recovery mechanisms.

The apparent activation energy for Y-junction migration in 2N Ni was estimated to be $260-300 \mathrm{~kJ} / \mathrm{mol}$, which is very close to that for recrystallization. Solutes suppress both Y-junction migration and recrystallization as corroborated by their apparent activation energies, but the latter is suppressed more than the former.

The addition of solutes promotes the strong pinning of $\mathrm{Y}$-junction migration via two important mechanisms: solute drag of medium/high angle lamellar GNBs and Y-junction pinning by dislocations present in low angle IDBs.

\section{Acknowledgements}

This project has received funding from the European Research Council (ERC) under the European Union's Horizon 2020 research and innovation programme (grant agreement No 788567). The authors are grateful to N. Hansen and D. Juul Jensen for valuable comments, and to Y.B. Zhang for providing the deformed $3 \mathrm{~N} \mathrm{Ni}$ samples and his unpublished raw data for the recrystallization/recovery analyses of $3 \mathrm{~N}$ Ni performed herein.

\section{Disclosure statement}

No potential conflict of interest. 


\section{References}

[1] D.A. Hughes, N. Hansen, Exploring the limit of dislocation based plasticity in nanostructured metals, Phys. Rev. Lett. 112 (2014), pp. 112:135504.

[2] T. Yu, N. Hansen, X. Huang, Linking recovery and recrystallization through triple junction motion in aluminum cold rolled to a large strain, Acta Mater. 61 (2013), 6577-6586.

[3] T. Yu, N. Hansen, X. Huang, Recovery by triple junction motion in aluminium deformed to ultrahigh strain, Proc. R. Soc. A. 467 (2011), pp. 3039-3065.

[4] T. Yu, N. Hansen, X. Huang, Recovery mechanisms in nanostructured aluminium, Philos. Mag. 92 (2012), pp. 4056-4074.

[5] D.A. Hughes, N. Hansen, Microstructure and strength of nickel at large strains, Acta Mater. 48 (2000), pp. 2985-3004.

[6] D.A. Hughes, N. Hansen, The microstructural origin of work hardening stages, Acta Mater. 148 (2018), pp. 374-383.

[7] Q. Liu, X. Huang, D.J. Lloyd, N. Hansen, Microstructure and strength of commercial purity aluminium (AA 1200) cold-rolled to large strains, Acta Mater. 50 (2002), pp. 3789-3802.

[8] H.W. Zhang, X. Huang, R. Pippan, N. Hansen, Thermal behavior of Ni (99.967\% and 99.5\% purity) deformed to an ultra-high strain by high pressure torsion, Acta Mater. 58 (2010), pp. 1698-1707.

[9] L. M. Clarebrough, M. E. Hargreaves, M. H. Loretto, Changes in internal energy associated with recovery and recrystallization, in Recovery and Recrystallization of Metals, L. Himmel, ed., Interscience Publishers, New York, 1963. pp. 63-121.

[10] Y.B. Zhang, O.V. Mishin, N. Kamikawa, A. Godfrey, W. Liu, Q. Liu, Microstructure and mechanical properties of nickel processed by accumulative roll bonding, Mater. Sci. Eng. A. 576 (2013), pp. 160-166.

[11] Y.B. Zhang, O. V. Mishin, A. Godfrey, Analysis of through-thickness heterogeneities of microstructure and texture in nickel after accumulative roll bonding, J. Mater. Sci. 49 (2014), pp. 287-293.

[12] G. Christiansen, J.R. Bowen, J. Lindbo, Electrolytic preparation of metallic thin foils with large electrontransparent regions, Mater. Charact. 49 (2002), pp. 331-335.

[13] A.R. Jones, B. Ralph, N. Hansen, Subgrain coalescence and the nucleation of recrystallization at grain boundaries in aluminium, Proc. R. Soc. London A 368 (1979), pp. 345-357. 
[14] T. Yu, N. Hansen, X. Huang, A. Godfrey, Observation of a new mechanism balancing hardening and softening in metals, Mater. Res. Lett. 2 (2014), pp. 160-165.

[15] T. Yu, N. Hansen, Coarsening kinetics of fine-scale microstructures in deformed materials, Acta Mater. 120 (2016), pp. 40-45.

[16] G. Borelius, S. Berglund, S. Sjoberg, Measurements on the evolution of heat during the recovery of coldworked metals, Ark. Fys. 6 (1952), pp. 143-149.

[17] O.V. Mishin, Y.B. Zhang, A. Godfrey. The influence of multiscale heterogeneity on recrystallization in nickel processed by accumulative roll bonding, J. Mater. Sci. 52 (2017) pp. 2730-2745.

[18] R.E. Cook, G. Gottstein, U.F. Kocks, Recovery in deformed copper and nickel single crystals, J. Mater. Sci. 18 (1983), pp. 2650-2664.

[19] P. Bartuska, Establishment of activation energy of subgrain growth in strongly deformed polycrystalline nickel, Czechoslov. J. Phys. 15 (1965), pp. 678-685.

[20] G. Neumann, C. Tuijn, Self-diffusion and impurity diffusion in pure metals: handbook of experimental data, Pergamon, Oxford, 2011.

[21] S. V. Divinski, G. Reglitz, G. Wilde, Grain boundary self-diffusion in polycrystalline nickel of different purity levels, Acta Mater. 58 (2010), pp. 386-395.

[22] T. Yu, D.A. Hughes, N. Hansen, X. Huang, In situ observation of triple junction motion during recovery of heavily deformed aluminum, Acta Mater. 86 (2015), pp. 269-278. 
Table 1. Chemical composition of the nickel used in the present study (wt.\%).

\begin{tabular}{llllllllllll}
\hline & $\mathrm{Ni}$ & $\mathrm{Fe}$ & $\mathrm{Mn}$ & $\mathrm{Si}$ & $\mathrm{C}$ & $\mathrm{Cu}$ & $\mathrm{S}$ & $\mathrm{P}$ & $\mathrm{Al}$ & $\mathrm{Mg}$ & $\mathrm{Ti}$ \\
\hline $2 \mathrm{~N} \mathrm{Ni}$ & 99.5 & 0.13 & 0.24 & 0.04 & 0.01 & 0.03 & 0.001 & - & - & - & - \\
& & & & & & & & & & & \\
$3 \mathrm{~N} \mathrm{Ni}$ & 99.97 & 0.009 & 0.002 & 0.005 & $<0.002$ & 0.001 & $<0.001$ & 0.004 & 0.003 & 0.003 & 0.002 \\
& & & & & & & & & & & \\
\hline
\end{tabular}

Table 2. Average lamellar GNB spacing $\left(d_{G N B}\right)$, average IDB spacing $\left(d_{I D B}\right)$, fraction of high angle boundaries $\left(f_{H A B}\right)$ and fraction of rolling texture components $\left(f_{C+S+B}\right)$ of deformed $2 \mathrm{~N}$ and $3 \mathrm{~N} \mathrm{Ni}$.

\begin{tabular}{|c|c|c|c|c|c|c|}
\hline & \multicolumn{3}{|c|}{$D_{G N B}(\mathrm{~nm})$} & \multirow{2}{*}{$\begin{array}{c}\boldsymbol{d}_{I D B}(\boldsymbol{\mu m}) \\
\text { TEM }\end{array}$} & \multirow{2}{*}{$\begin{array}{c}f_{H A B}(\%) \\
E B S D\end{array}$} & \multirow{2}{*}{$\begin{array}{c}f_{C+S+B}(\%) \\
\text { EBSD }\end{array}$} \\
\hline & TEM & ECC & EBSD & & & \\
\hline $2 \mathrm{~N} \mathrm{Ni}$ & 83 & 105 & 110 & 0.2 & 57 & 86 \\
\hline 3N Ni & 124 & - & 160 & 0.3 & 50 & 84 \\
\hline
\end{tabular}


Figure 1. Deformation microstructures in the longitudinal sections of Ni compared to heavily cold-rolled Al. (a) Overview montages of $2 \mathrm{~N} \mathrm{Ni}$; (b) $3 \mathrm{~N} \mathrm{Ni}$; (c,d) TEM micrograph of Al and corresponding sketch of lamellar GNBs (black) and short IDBs (grey) (adapted from [3]). All triple junctions formed by lamellar GNBs (i.e. Y-junctions) in (d) are marked by arrows.
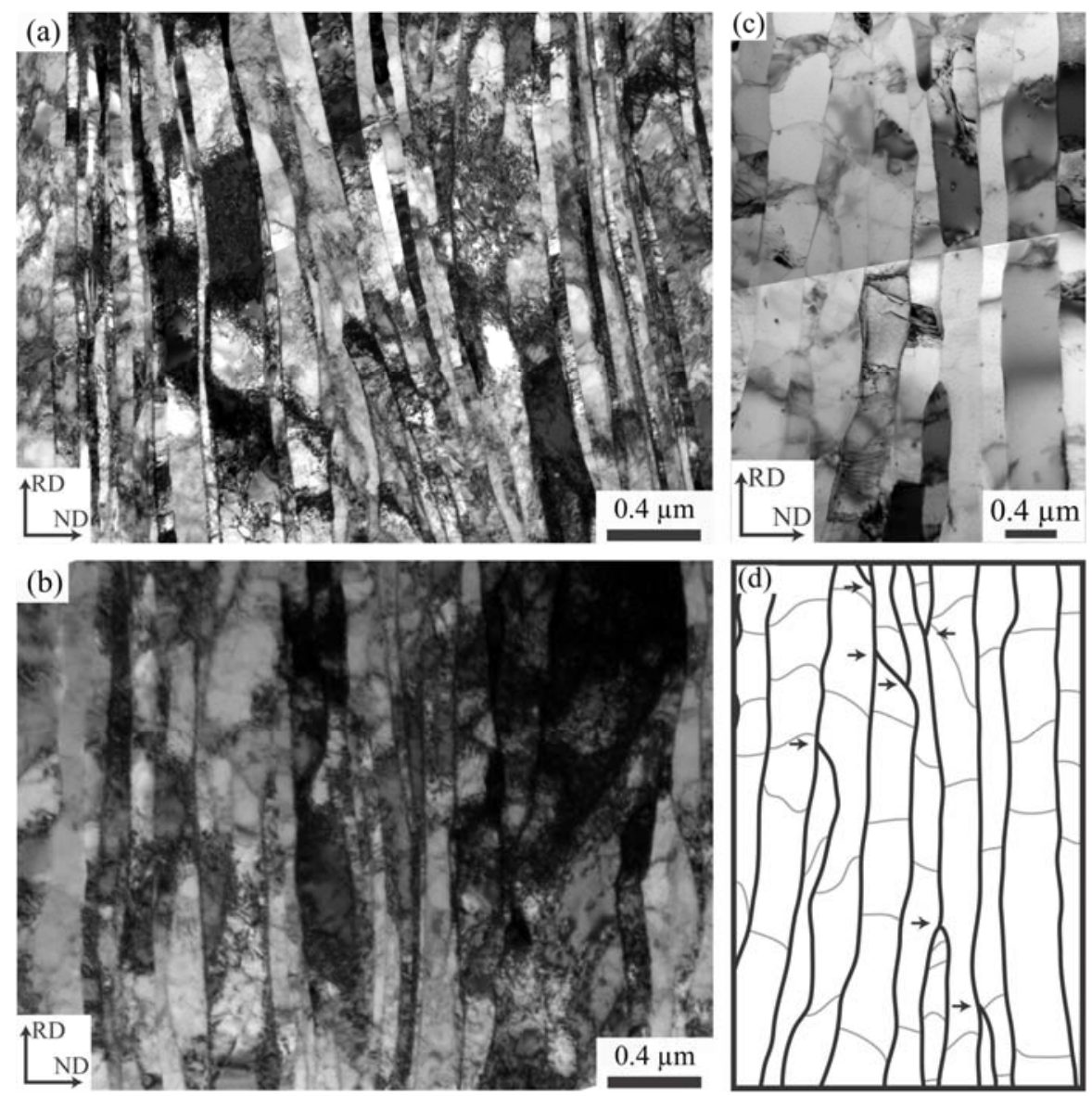
Figure 2. IDBs and interior dislocations within individual lamellae of deformed $2 \mathrm{~N} \mathrm{Ni}$. (a) Thin lamellae; (b) thick lamellae.


Figure 3. Y-junctions are pinned by IDBs and individual dislocations in 2N Ni. (a) TEM micrograph; (b) Tracing indicates lamellar boundaries (thick lines) and IDBs (grey stylized nets).

Figure 4. Examples of Y-junction pairs created by shearing in $2 \mathrm{~N} \mathrm{Ni}$. $(a, c)$ TEM micrographs; $(b, d)$ corresponding tracings of lamellar GNBs. The IDBs are not traced.
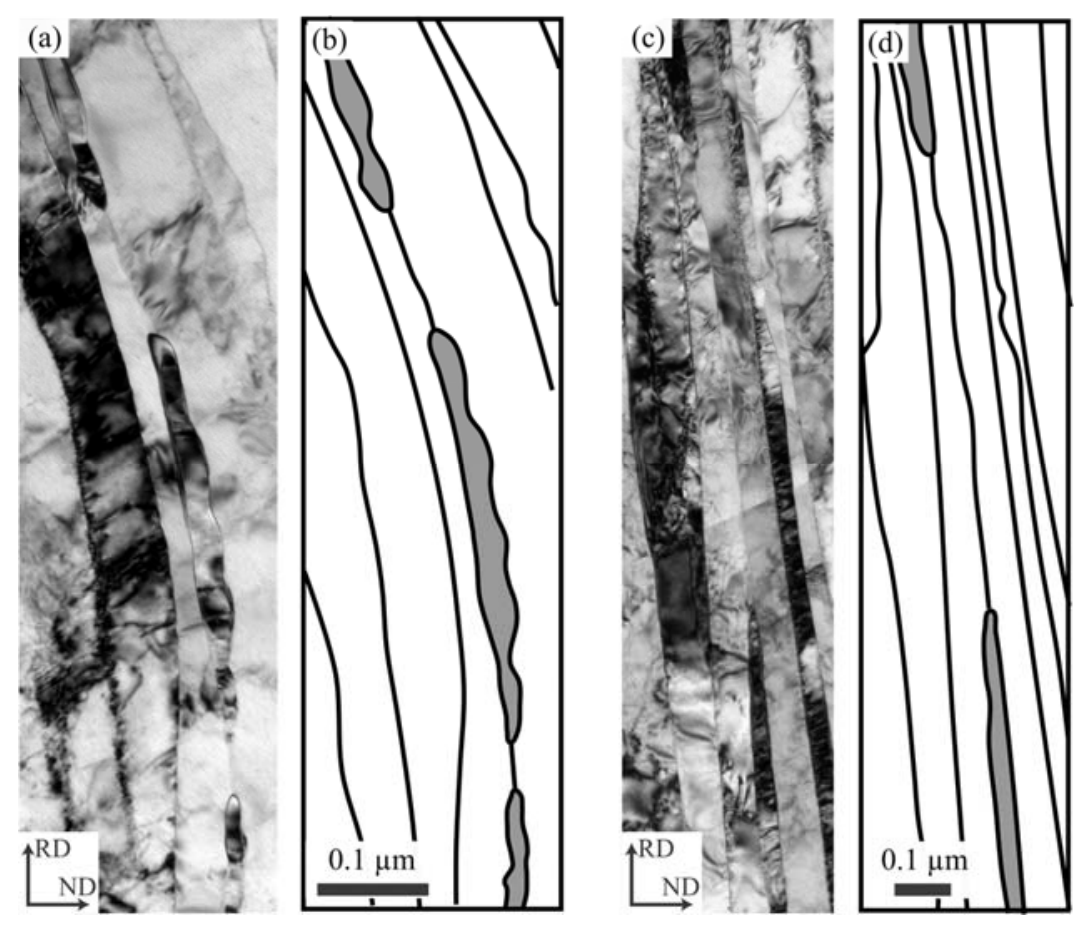

Figure 5. Hardness variation of $3 \mathrm{~N}$ and $2 \mathrm{~N}$ Ni during isochronal annealing $(1 \mathrm{~h})$. The error bars (in most cases smaller than the symbols) show the standard deviations.

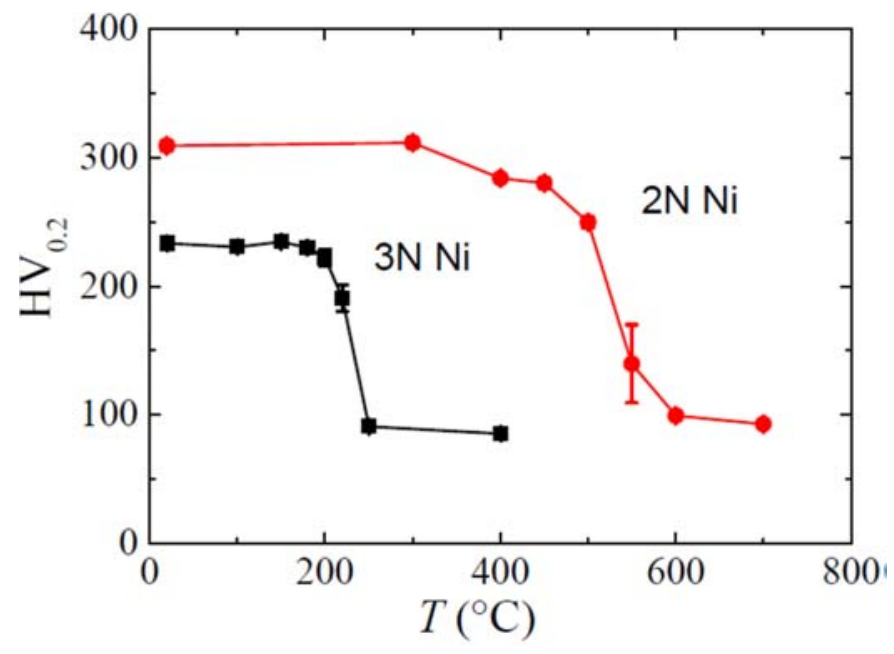


Figure 6. In situ TEM observation of Y-junction migration in $2 \mathrm{~N}$ Ni during annealing. (a) From 20 to $300^{\circ} \mathrm{C}$; (b) from 350 to $400^{\circ} \mathrm{C}$. The annealing times are given on the micrographs. The position of the shortening lamella is traced and shaded for each of the corresponding 5 states while the position of the tailing boundary indicates the initial state. The IDBs are not traced.
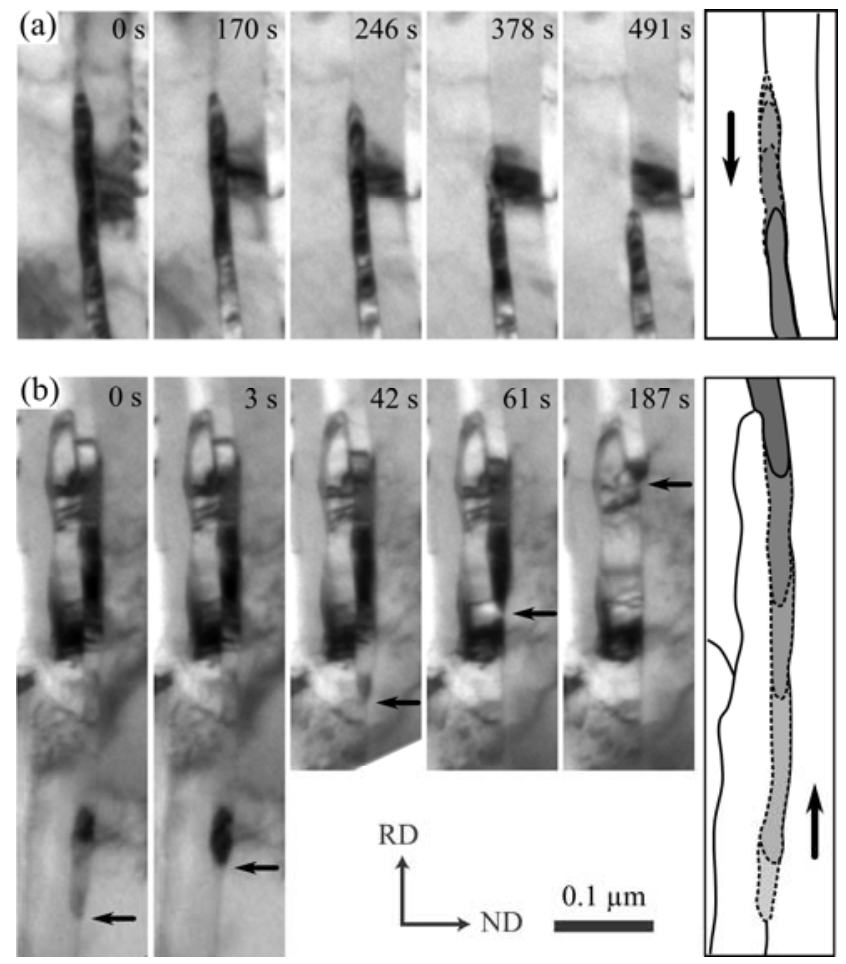

Figure 7. EBSD raw data showing Y-junction migration during ex situ annealing of $3 \mathrm{~N} \mathrm{Ni}$ at $200{ }^{\circ} \mathrm{C}$. (a) Before annealing; (b) after annealing for $1 \mathrm{~h}$; (c) tracing of the lamellar GNBs. The microstructure is colour coded according to the orientation of the transverse direction (TD), except that white pixels represent not-indexed regions. The black arrows indicate mobile $\mathrm{Y}$-junctions whose migration path is coloured (c).

(a)

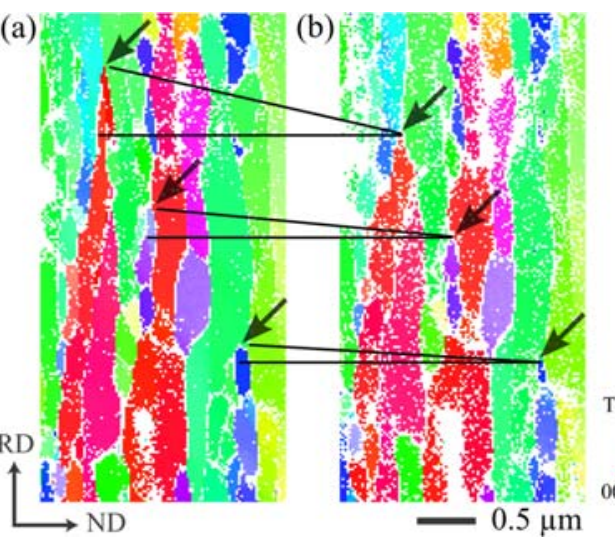

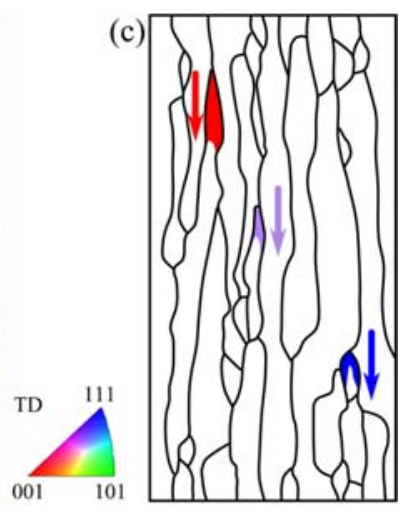


Figure 8. ECC images showing lamellar microstructures in the longitudinal section of $2 \mathrm{~N} \mathrm{Ni}$. (a) Deformed state; (b) $400{ }^{\circ} \mathrm{C}$ for $120 \mathrm{~h}$; (c) $450^{\circ} \mathrm{C}$ for $17 \mathrm{~h}$; (d) $500^{\circ} \mathrm{C}$ for $1 \mathrm{~h}$.
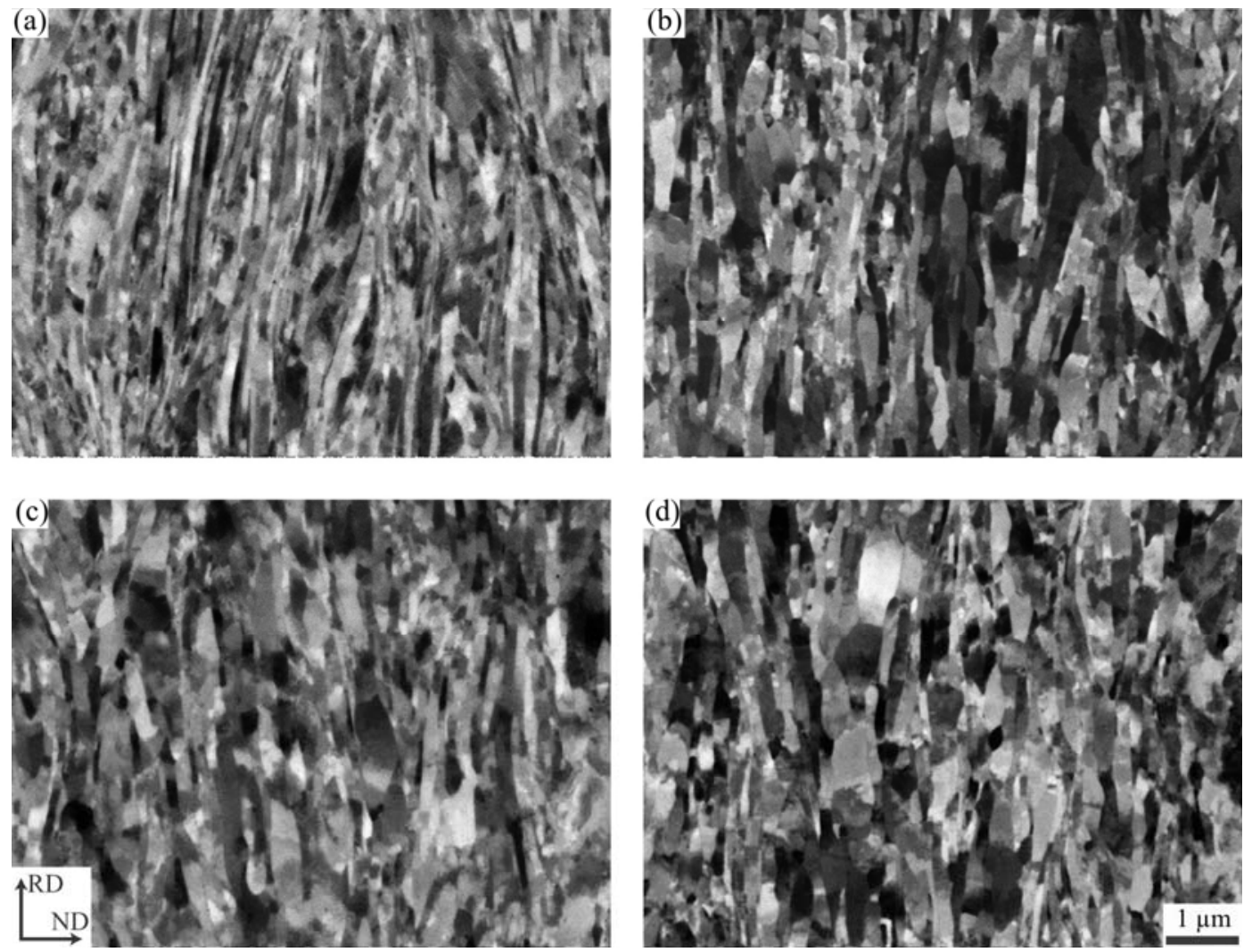

Figure 9. ECC images showing nucleation of recrystallization in $2 \mathrm{~N} \mathrm{Ni}$. (a) Annealed at $450^{\circ} \mathrm{C}$ for $17 \mathrm{~h}$; (b) $500{ }^{\circ} \mathrm{C}$ for $1 \mathrm{~h}$. The micron-sized nuclei are marked.
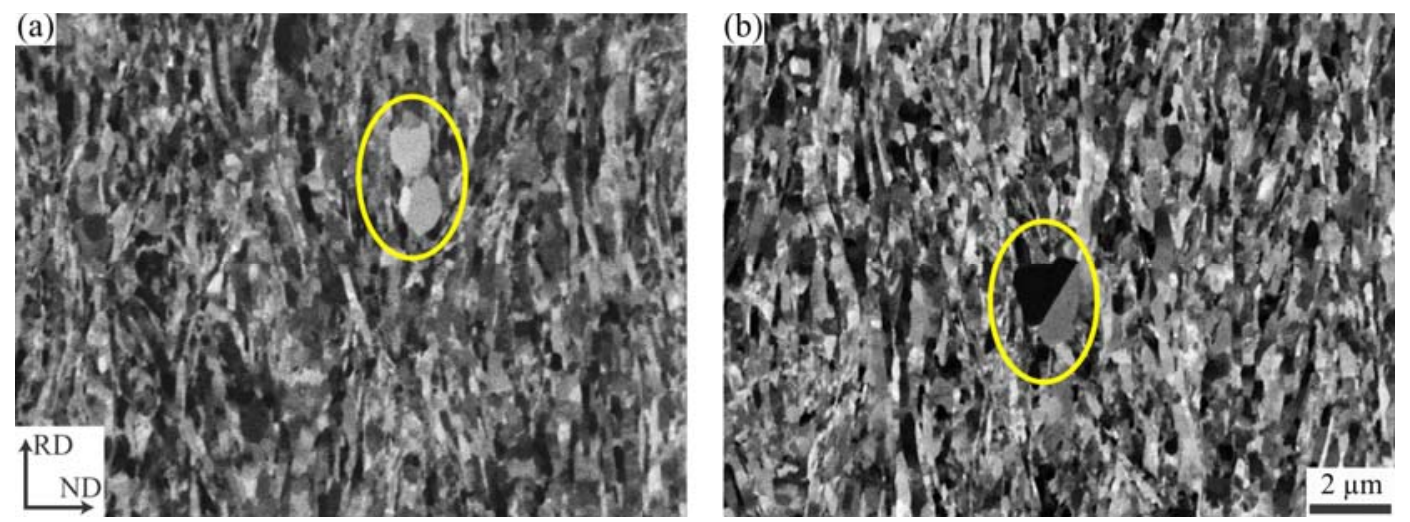
Figure 10. Recovery coarsening by $\mathrm{Y}$-junction migration in $2 \mathrm{~N} \mathrm{Ni}$. (a) Microstructure after annealing at $500^{\circ} \mathrm{C}$ for $0.5 \mathrm{~h}$; (b) EBSD (solid symbols) and ECC (open symbols) data for the average lamellar GNB spacing at different annealed states. Curves are fitted based on the model in Section 3.6.
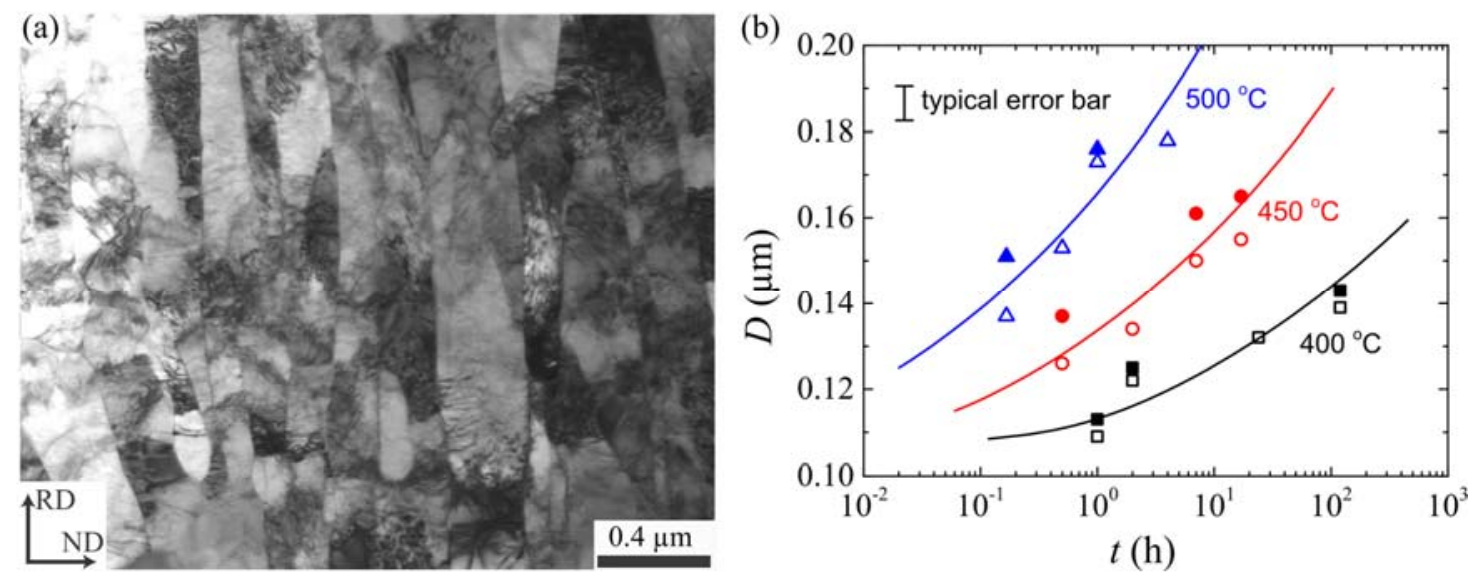

Figure 11. Coarsening and recrystallization nuclei formed in $3 \mathrm{~N} \mathrm{Ni}$ after annealing at $220^{\circ} \mathrm{C}$ for just 10 minutes. The microstructure is colour coded according to the orientation of the TD. High and low angle boundaries are shown in thick and thin lines, respectively.

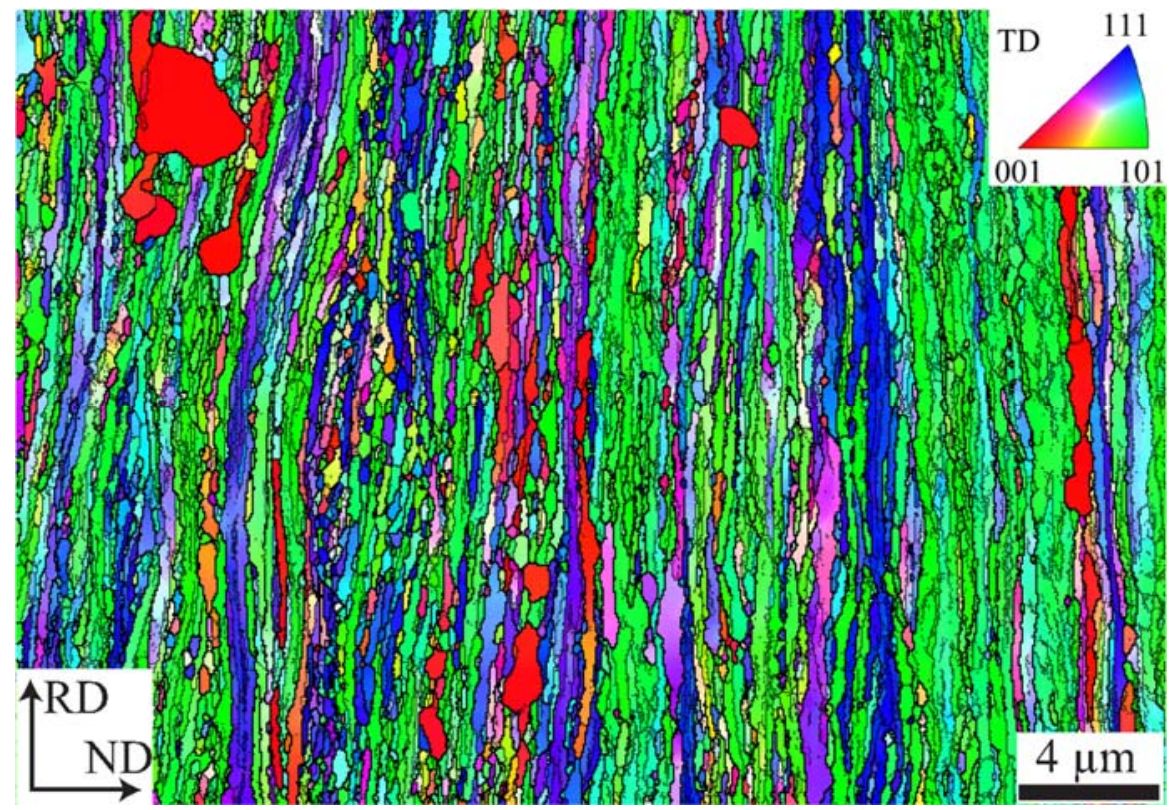


Figure 12. The increase of apparent activation energy during coarsening of $2 \mathrm{~N} \mathrm{Ni}$. The inset shows the determination of $Q_{0}$. Grey area is a model extrapolation at later stages.

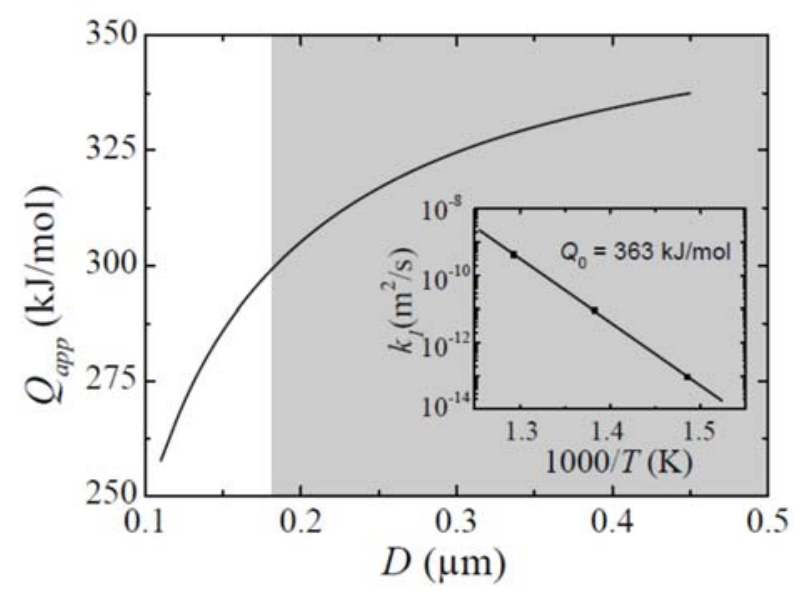

Figure 13. Recrystallization kinetics and apparent activation energies: $(a, b) 2 \mathrm{~N}$ Ni measured between 500 and $600{ }^{\circ} \mathrm{C} ;(\mathrm{c}, \mathrm{d}) 3 \mathrm{~N}$ Ni measured between 200 and $240^{\circ} \mathrm{C}$.
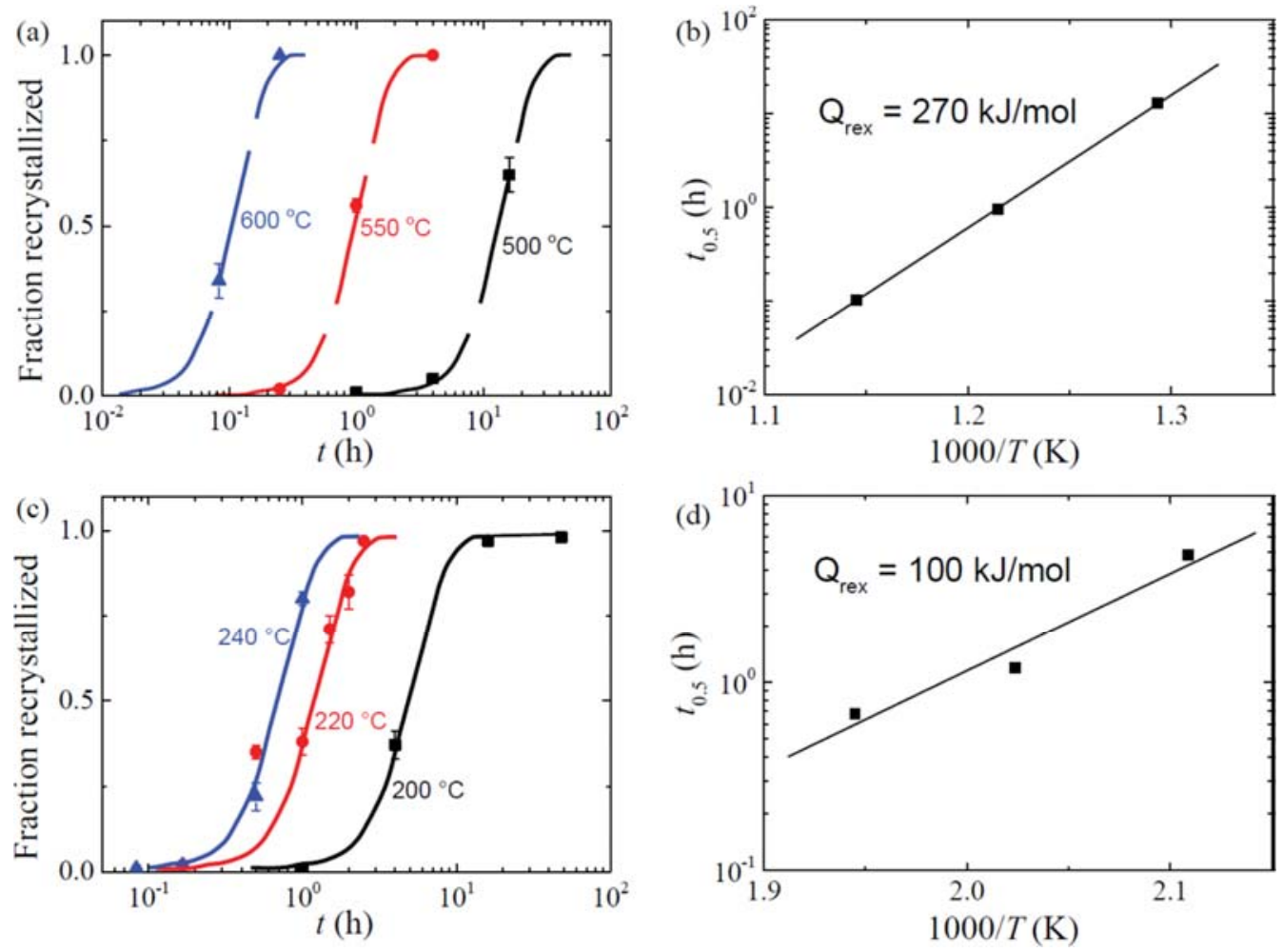
Figure 14. Distributions of the lamellar GNB spacing for deformed and annealed 2N Ni samples.

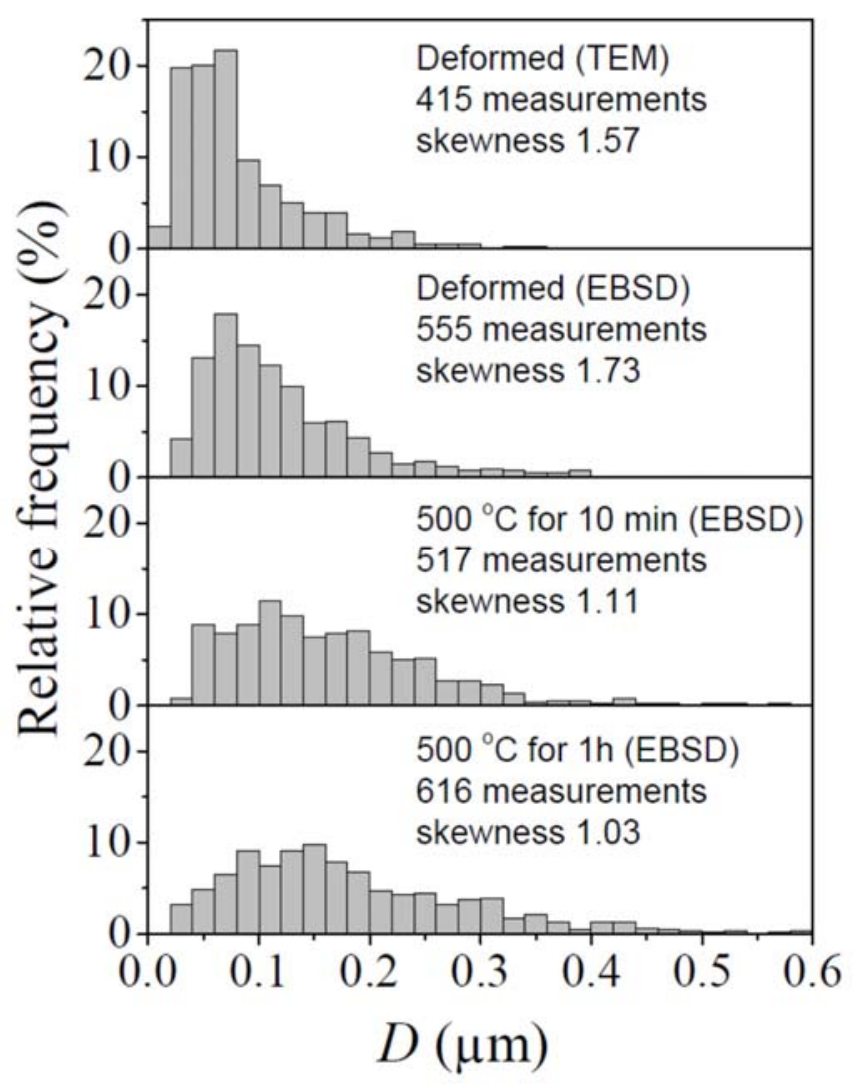

Figure 15. Comparison of coarsening for $2 \mathrm{~N} \mathrm{Ni}$ and $2 \mathrm{~N} \mathrm{Al}$ at the homologous temperature of 0.42 . The data for $2 \mathrm{~N} \mathrm{Ni}$ were measured by EBSD (solid symbols) and ECC (open symbols); the curve for $2 \mathrm{~N} \mathrm{Al}$ is predicted according to the coarsening model in [13].



